\title{
THE LIMITING BEHAVIOR OF THE KOBAYASHI-ROYDEN PSEUDOMETRIC
}

\author{
SHULIM KALIMAN
}

\begin{abstract}
We study the limit of the sequence of Kobayashi metrics of Riemann surfaces (when these Riemann surfaces form an analytic fibration in such a way that the total space of fibration becomes a complex surface), as the fibers approach the center fiber which is not in general smooth. We prove that if the total space is a Stein surface and the smooth part of the center fiber contains a component biholomorphic to a quotient of the disk by a Fuchsian group of first kind, then the Kobayashi metrics of the near-by fibers converge to the Kobayashi metric of this component as fibers tend to the center fiber.
\end{abstract}

\section{INTRODUCTION}

Let $\Phi: M \rightarrow \Delta$ be a holomorphic mapping from a complex surface $M$ on the disc $\Delta=\{z \in C|| z \mid<1\}$. Suppose that for each $c \neq 0 \Gamma_{c}=\Phi^{-1}(c)$ is a smooth noncompact Riemann surface and $\Gamma_{0}^{*}$ is a smooth part of $\Gamma_{0}=\Phi^{-1}(0)$. We shall investigate relations between the Kobayashi-Royden pseudometric $k_{\Gamma_{0}^{*}}$ on $\Gamma_{0}^{*}$ and the limit of the Kobayashi-Royden pseudometric on nearby fibers. More precisely, we shall study the problem when the equality

$$
\lim _{c \rightarrow 0} k_{\Gamma_{c}}=k_{\Gamma_{0}^{*}}
$$

holds. In general, it is not so. In [PS, §2.2] there is an example of such mapping $\Phi: M \rightarrow \Delta$, where $M$ is a holomorphically convex region in $C^{2}$, every $\Gamma_{c}$ is a disc, but $\lim _{c \rightarrow 0} k_{\Gamma_{c}} \neq k_{\Gamma_{0}}$. Zaidenberg found certain sufficient conditions, which imply (1) [Z]. But his result does not give the answer to the question whether (1) holds, when $\Phi$ is a polynomial of two complex variables and $M=$ $\Phi^{-1}(\Delta)$. He supposed that the answer was positive. Let $G$ be a Fuchsian group of the first kind. The Main Theorem of this paper says that, if $M$ is a Stein surface and $\Gamma_{0}^{*}$ contains a component $R$, which is biholomorphically equivalent to $\Delta / G$, then $\lim _{c \rightarrow 0} k_{\Gamma_{c}}=k_{R}$. In particular, the Zaidenberg's conjecture is true. The last fact was announced in [Ka], where it was used to classify isotrivial polynomials on $C^{2}$.

The paper is organized as follows. We present some terminology and formulate our main results in the first section. The second section contains a technical lemma about Fuchsian groups and its corollaries needed for the proof of the Main Theorem. This lemma asserts that two noncommutative nonelliptic elements of a Fuchsian group cannot move any point $z \in \Delta$ by a distance less than

Received by the editors December 13, 1990 and, in revised form, June 21, 1991.

1980 Mathematics Subject Classification (1985 Revision). Primary 32H15. 
a certain $\varepsilon>0$ at the same time. Next we handle the case of hyperbolic fibers $\left\{\Gamma_{b_{j}}\right\}$ with $b_{j} \rightarrow 0$. We consider universal holomorphic covering $f_{j}: \Delta \rightarrow \Gamma_{b_{j}}$ and find out when $\left\{f_{j}\right\}$ converge to an unramified mapping $f: D \rightarrow \Gamma_{0}^{*}$ on a certain maximal region $D \subset \Delta$. We also prove that $f(D)$ is a component of $\Gamma_{0}^{*}$ and, if $D=\Delta$, then the Kobayashi-Royden pseudometric on $f(D)$ coincides with the limit of the Kobayashi-Royden pseudometric of nearby fibers. The result of the forth section says that $D$ is simply connected in the case when $M$ is a Stein surface. The last section contains the proof of the Main Theorem.

It is a great pleasure to thank M. G. Zaidenberg for his question and stimulating discussions.

\section{Formulation OF THE MAIN THEOREM}

First we fix terminology, notations and definitions that we shall use throughout the paper. Every manifold we are going to consider will be complex. If $Y$ is a manifold, then $T Y$ is its holomorphic tangent bundle and $T_{y} Y$ is a tangent space at a point $y \in Y$. Put $\Delta_{r}=\{z \in C|| z \mid<r\}, \Delta=\Delta_{1}$, and $\Delta^{*}=\Delta-0$. By a curve $\eta$ in $Y$ we mean a continuous mapping $\eta:[0,1] \rightarrow Y$. A loop $\gamma$ in $Y$ is a curve with $\gamma(0)=\gamma(1)$. In other words, $\gamma$ is a continuous mapping from $\partial \Delta$ to $Y$ (as is frequently done, we use the symbol $\partial$ to denote boundaries). If $x \in \gamma(\partial \Delta)$, then we write $x \in \gamma$. Recall that a differential pseudometric on a complex manifold $Y$ is a nonnegative homogeneous function on the tangent bundle $T Y$, i.e., it is a function $p: T Y \rightarrow \mathbf{R}$ such that $p(y, v) \geq 0, p(y, \lambda v)=|\lambda| p(y, v)$ for all $y \in Y, v \in T_{y} Y$, and $\lambda \in C$. When $p$ is continuous, we call the pseudometric continuous. If $Y$ is connected and for each piecewise smooth curve $\eta$ in $Y$ there exists the integral $P(\eta)=\int_{0}^{1} p(\eta(t), d \eta(t)) d t$, one can define the integral pseudometric $P(x, y)=\inf _{\eta}\{P(\eta) \mid \eta(0)=x, \eta(1)=y\}$. Of course, the integral pseudometric exists, when a proper differential pseudometric is continuous. The KobayashiRoyden differential pseudometric is given by the formula

$$
k_{Y}(y, v)=\inf _{r}\left\{1 / r \mid \phi \in \operatorname{Hol}\left(\Delta_{r}, Y\right), \phi(0)=y, d \phi(0)=v\right\} .
$$

By Royden's theorem [R] it generates the integral pseudometric $K_{Y}$ which coincides with the Kobayashi pseudometric on $Y$ [Ko].

Throughout the paper $\Phi: M \rightarrow \Delta$ is a holomorphic mapping from a smooth complex surface $M$ on $\Delta$ such that for $c \neq 0 \quad \Gamma_{c}=\Phi^{-1}(c)$ is a smooth Riemann surface. We shall say that $\Phi: M \rightarrow \Delta$ is a family of Riemann surfaces. The fiber $\Gamma_{0}=\Phi^{-1}(0)$ can contain singular points. Denote the smooth part of $\Gamma_{0}$ by $\Gamma_{0}^{*}$. Let $\beta=\left\{b_{j}\right\} \subset \Delta^{*}$ be a sequence that tends to zero, let $R$ be a component of $\Gamma_{0}^{*}$. We say that $\lim _{j \rightarrow \infty} k_{\Gamma_{b_{j}}}=k_{R}\left(\right.$ or $\left.\varlimsup_{j \rightarrow \infty} k_{\Gamma_{b_{j}}} \leq k_{R}\right)$, if for each sequence $\left\{w_{j} \in T \Gamma_{b_{j}}\right\}$ that converges to $w \in T R$ in the topology of $T M$ the equality $\lim _{j \rightarrow \infty} k_{\Gamma_{b_{j}}}\left(w_{j}\right)=k_{R}(w)$ (or inequality $\overline{\lim }_{j \rightarrow \infty} k_{\Gamma_{b_{j}}}\left(w_{j}\right) \leq$ $\left.k_{R}(w)\right)$ holds. If $\lim _{j \rightarrow \infty} k_{\Gamma_{b_{j}}}=k_{R}$ for each sequence $\beta$ as above, then we say $\lim _{c \rightarrow 0} k_{\Gamma_{c}}=k_{R}$. In the same meaning $\varlimsup_{c \rightarrow \infty} k_{\Gamma_{c}} \leq k_{R}$. The following two results belong to Zaidenberg $[\mathrm{Z}]$.

Proposition 1.1. For each component $R$ of $\Gamma_{0}^{*}$ the inequality $\varlimsup_{c \rightarrow \infty} k_{\Gamma_{c}} \leq k_{R}$ holds. 
Theorem 1.2. Let $\bar{M}$ be a smooth compact surface and $\bar{\Gamma} \subset \bar{M}$ be an analytic curve in $\bar{M}$. Suppose that $M \subset \bar{M}-\bar{\Gamma}, \bar{\Gamma}_{0}=\overline{\bigcap_{r>0} \Phi^{-1}\left(\Delta_{r}\right)}$, and $\Gamma_{0}=\bar{\Gamma}_{0}-\bar{\Gamma}$. If every component of $\Gamma_{0}^{*}$ is hyperbolic, then $\lim _{c \rightarrow 0} k_{\Gamma_{\mathrm{c}}}=k_{R}$.

Zaidenberg conjectured that, if $\Phi$ is a polynomial on $C^{2}$ and $M=\Phi^{-1}(\Delta)$, then the assumption that all the components of $\Gamma_{0}^{*}$ are hyperbolic can be omitted. We shall show that this hypothesis is correct. Recall that $G$ is a Fuchsian group of the first kind, if the closure of the orbit $\{g(0) \mid g \in G\}$ in $C$ contains $\partial \Delta$ [B]. In particular, in the polynomial case every hyperbolic component $R$ of $\Gamma_{0}^{*}$ has a representation $R \cong \Delta / G$, where $G$ is a Fuchsian group of the first kind.

Main Theorem. Let $\Phi: M \rightarrow \Delta$ be a family of Riemann surfaces. Suppose that $M$ is a Stein manifold and $\Gamma_{0}^{*}$ contains a component $R$ that is biholomorphically equivalent to $\Delta / G$, where $G$ is a Fuchsian group of the first kind. Then $\lim _{c \rightarrow 0} k_{\Gamma_{c}}=k_{R}$.

Note that, if $R$ is nonhyperbolic, such a fact follows from Proposition 1.1. Hence we have

Corollary. Let $\Phi: C^{2} \rightarrow C$ be a polynomial. Then $\lim _{c \rightarrow 0} k_{\Gamma_{c}}=k_{\Gamma_{0}^{*}}$.

We shall restrict ourselves to the case of connected fibers for $c \neq 0$ (in general case the proof is the same, but instead of $\Gamma_{c}$ we have to use their components).

\section{ONE PROPERTY OF FUCHSIAN GROUPS}

We shall denote the Kobayashi metric on $\Delta$ by $K_{\Delta}$.

Lemma 2.1. For every $r>0$ there exists $\varepsilon>0$ such that for every Fuchsian group $G$, noncommutative elements $a^{\prime}, b^{\prime} \in G$, and a point $z \in \Delta$ satisfying $0<K_{\Delta}\left(z, a^{\prime}(z)\right)<r$, either $K_{\Delta}\left(z, b^{\prime}(z)\right)>\varepsilon$ or $z$ is a fixed point of the mapping $b^{\prime}: \Delta \rightarrow \Delta$.

Proof. Assume, to reach a contradiction, that for a certain $r>0$ and each $\varepsilon>0$ there exists a Fuchsian group $G_{\varepsilon}$, noncommutative elements $a_{\varepsilon}^{\prime}, b_{\varepsilon}^{\prime} \in G_{\varepsilon}$, and a point $z_{\varepsilon} \in \Delta$ such that $0<K_{\Delta}\left(z_{\varepsilon}, a_{\varepsilon}^{\prime}\left(z_{\varepsilon}\right)\right)<r$ and $0<K_{\Delta}\left(z_{\varepsilon}, b_{\varepsilon}^{\prime}(z)\right)<\varepsilon$. We shall show that for a sufficiently small $\varepsilon$ the group $G_{\varepsilon}$ cannot be discontinuous. Without loss of generality, we set $z_{\varepsilon}=0$. Let id be the identity element of $G_{\varepsilon}$. Since $G_{\varepsilon}$ is a discontinuous group, one can find elements $a_{\varepsilon}$ and $b_{\varepsilon}$ satisfying

$$
\begin{gathered}
K_{\Delta}\left(0, b_{\varepsilon}(0)\right)=\min \left\{K_{\Delta}(0, g(0)) \mid g \in G_{\varepsilon}, g(0) \neq 0\right\}, \\
K_{\Delta}\left(0, a_{\varepsilon}(0)\right)=\min \left\{K_{\Delta}(0, g(0)) \mid g \in G_{\varepsilon}, g(0) \neq 0,\left[b_{\varepsilon}, g\right] \neq \mathrm{id}\right\} .
\end{gathered}
$$

The mapping $a_{\varepsilon}$ and $b_{\varepsilon}$ can be represented in the form

$$
\begin{array}{lll}
a_{\varepsilon}(z)=e^{i \theta_{\varepsilon}}\left(z+\alpha_{\varepsilon}\right) /\left(1+\bar{\alpha}_{\varepsilon} z\right), & & \left|\theta_{\varepsilon}\right| \in[0, \pi], \\
b_{\varepsilon}(z)=e^{i \tau_{\varepsilon}}\left(z+\beta_{\varepsilon}\right) /\left(1+\bar{\beta}_{\varepsilon} z\right), & & \left|\tau_{\varepsilon}\right| \in[0, \pi] .
\end{array}
$$

We shall omit the index $\varepsilon$ from now on, if it does not cause misunderstanding. Let us consider $b$ as a function of two variables $z$ and $\beta$. Expand $b$ in power series of $z, \beta$, and $\bar{\beta}$. Then $b(z)=e^{i \tau} z+e^{i \tau} \beta$ up to the nonlinear terms. Hence for every natural $m$ one can find a neighborhood of the origin 
in $C^{2}=\{(z, \beta)\}$ so that for all $n=1,2, \ldots, m$,

$$
b^{n}(z)=e^{i n \tau} z+\sum_{l=1}^{n} e^{i l \tau} \beta+O\left(|z|^{2}+|\beta|^{2}\right)
$$

in this neighborhood. Thus

$$
b^{n}(0)=\beta \sum_{l=1}^{n} e^{i l r}+O\left(|\beta|^{2}\right)=\beta e^{i n \tau}\left(e^{i n \tau}-1\right) /\left(e^{i \tau}-1\right)+O\left(|\beta|^{2}\right) .
$$

It is easy to check that for each $\tau_{0} \neq 2 \pi k$ there is a neighborhood $U$ of $\tau_{0}$ and integer $n \geq 2$ so that for every $\tau \in U$,

$$
\left|\left(e^{i n \tau}-1\right) /\left(e^{i \tau}-1\right)\right|<1 .
$$

Now one can see that $\tau_{\varepsilon} \rightarrow 0$ as $\varepsilon \rightarrow 0$. Indeed, the preceding assumption implies

$$
0<|\alpha|<\tilde{r}, \quad 0<|\beta|<\tilde{\varepsilon},
$$

where $\tilde{\varepsilon}=\left(e^{\varepsilon}+1\right)$ and $\tilde{r}=\left(e^{r}-1\right) /\left(e^{r}+1\right)$. Thus $\lim _{\varepsilon \rightarrow 0}\left|\beta_{\varepsilon}\right|=0$. Assume $\overline{\lim }_{\varepsilon \rightarrow 0}\left|\tau_{\varepsilon}\right|>1 / m$. Then by (2.3) we can find $n \leq m$ with $\left|b^{n}(0)\right|<|b(0)|$. This contradicts (2.1). Thus $b_{\varepsilon}(z) \rightarrow z$ uniformly on compact subsets of $\Delta$ as $\varepsilon \rightarrow 0$. Let $\varlimsup_{\overline{\lim \rightarrow 0}}\left|\alpha_{\varepsilon}\right|=\alpha^{0}$. Since $\left|\alpha_{\varepsilon}\right|<\tilde{r}, a_{\varepsilon} \circ b_{\varepsilon} \circ a_{\varepsilon}^{-1}(z) \rightarrow z$ as $\varepsilon \rightarrow 0$. In particular, for any sufficiently small $\varepsilon$ we have $\left|a_{\varepsilon} b_{\varepsilon} a_{\varepsilon}^{-1}(0)\right|<\alpha^{0} / 2$. This implies either $\alpha^{0}=0$ or $b$ and $a b a^{-1}$ are commutative. We shall prove that the last case does not hold. One can represent $a$ and $b$ as mappings of the upper half-plane. Then, if $a$ is a hyperbolic element, we may put $a(z)=\lambda z$ with $\lambda>0$ and if $a$ is a parabolic element, we may put $a(z)=z+1$ [A]. In both cases for any $b(z)=(p z+q) /(t z+s)$ with $p, q, t, s \in \mathbf{R}$ the direct computation shows that $\left[a b a^{-1}, b\right]=\mathrm{id}$, iff $[a, b]=\mathrm{id}$. When $a$ is an elliptic element, one may consider $a$ as a mapping $a: \Delta \rightarrow \Delta$ given by the formula $a(z)=\lambda z$ with $\lambda^{n}=1$ for a certain natural $n$. Again it is easy to show that $\left[a b a^{-1}, b\right]=\mathrm{id}$, iff $[a, b]=\mathrm{id}$ for any Möbius transformation $b: \Delta \rightarrow \Delta$. But this a contradicts (2.2). Therefore $\lim _{\varepsilon \rightarrow 0} \alpha_{\varepsilon}=0$. Same arguments as above show that $\theta_{\varepsilon} \rightarrow 0$ as $\varepsilon \rightarrow 0$. Hence for any sufficiently small $\varepsilon$ we have $\left|e^{i \theta_{\varepsilon}}-1\right|+\left|e^{i \tau_{\varepsilon}}-1\right|<1 / 2$ and for an arbitrarily small $\alpha$ the following inequality holds

$$
\left|b^{-1} a^{-1} b a(0)\right| \approx\left|e^{i \tau}-1\right||\alpha|+\left|e^{i \theta}-1\right||\beta|<|\alpha| / 2<|a(0)|
$$

but $b^{-1} a^{-1} b a$ and $b$ are not commutative, since $\left[a^{-1}, b a, b\right] \neq \mathrm{id}$. This is a contradiction.

Corollary 2.2. For every $r>0$ there exists $\varepsilon>0$ such that for every hyperbolic Riemann surface $R$, for every point $x \in R$, and for every couple of loops $\gamma$ and $\mu$ that generate noncommutative elements of the fundamental group $\pi_{1}(R, x)$, the inequalities $K_{R}(\gamma)<\varepsilon$ and $K_{R}(\mu)<r$ do not hold simultaneously.

The next three lemmas enable us to restate this corollary in a form which will be convenient for our following needs.

Lemma 2.3. Let $\gamma$ be a noncontractible loop on a Riemann surface $R$. Suppose that the corresponding element of the fundamental group $\pi_{1}(R)$ has a representation $[\gamma]=[\mu]^{n}$, where $[\mu] \in \pi_{1}(R)$ and the natural number $n \geq 2$. Then $\gamma$ has points of self-intersection. 
Proof. Let $H$ be the upper half-plane, and let $f: H \rightarrow R$ be a universal holomorphic covering. Then we can define the Möbius transformation $b: H \rightarrow H$ corresponding to $[\mu]$. If $b$ is a hyperbolic transformation, one can choose $f$ so that $b(z)=\lambda z$ with $\lambda>0[\mathrm{~A}]$. Let $z_{0}$ be a point in the inverse image of a point $x_{0} \in \gamma$. Obviously, each curve in $H$ that connects the points $z_{0}$ and $\lambda^{n} z_{0}$ contains points $z^{\prime}$ and $z^{\prime \prime}$ such that $z^{\prime}=\lambda z^{\prime \prime}$. But this means that $\gamma$ has the point of self-intersection $f\left(z^{\prime}\right)$. If $b$ is parabolic, we may suppose that $b(z)=z+1$. Again each curve that connects the points $z_{0}$ and $z_{0}+n$ contains points $z^{\prime}$ and $z^{\prime \prime}=z^{\prime}+1$. This implies the desired conclusion.

Lemma 2.4. Let $\gamma$ and $\mu$ be disjoint noncontractible loops in a Riemann surface $R$. Suppose that neither $\gamma$ nor $\mu$ has points of self-intersection. Then $\gamma$ and $\mu$ are homotopically equivalent, iff there is a region $U \subset R$ such that $\partial U=\gamma \cup \mu$ and $U$ is topologically an annulus.

Proof. Let $x_{1} \in \gamma$ and $y_{1} \in \mu$. Choose a curve $\nu_{1}:[0,1] \rightarrow R$ so that $\nu_{1}(0)=x_{1}, u_{1}(1)=y_{1}, \nu_{1}$ has no points of self-intersection and $\nu_{1}$ intersects $\gamma \cup \mu$ at the points $x_{1}$ and $y_{1}$ only. Choose an analogous curve $\nu_{2}$ so that $\nu_{2}$ connects points $x_{2} \in \gamma$ and $y_{2} \in \mu$, and $\nu_{2}$ is sufficiently close to, but disjoint from $\nu_{1}$. Then $\gamma-\left(x_{1} \cup x_{2}\right)$ consists of two components $\gamma_{1}$ and $\gamma_{2}$, and $\gamma_{1}$ is small enough. In the same way $\mu-\left(y_{1} \cup y_{2}\right)=\mu_{1} \cup \mu_{2}$, and $\mu_{1}$ is small. Then there exists an open disc $D \subset R$ with $\partial D=\nu_{1} \cup \nu_{2} \cup \gamma_{1} \cup \mu_{1}$. One can construct the loop $\eta=\nu_{1} \cup \mu_{2} \cup \nu_{2} \cup \gamma_{2}$. Since $\gamma$ and $\mu$ are homotopically equivalent, $\eta$ must be contractible. By our construction $\eta$ has no points of self-intersection. This implies the existence of the disc $U \subset R$ with $\partial U=\eta$. If $U \supset D$, then $U-\bar{D}$ contains the two components $U_{1}$ and $U_{2}$. Each of them is a disc, $\partial U_{1}=\gamma$ and $\partial U_{2}=\mu$. This contradicts the assumption that $\gamma$ and $\mu$ are noncontractible. Hence $U \cap D=\varnothing$. Obviously, $\bar{D} \cup \bar{U}$ is topologically a closed annulus and $\partial(\bar{U} \cup \bar{D})=\gamma \cup \mu$. This completes the proof of the lemma.

Lemma 2.5. Let $\gamma$ and $\mu$ be noncontractible loops on a Riemann surface $R$, and neither $\gamma$ nor $\mu$ has points of self-intersection. Suppose that $R-(\gamma \cup \mu)$ does not contain components that are topologically an annulus. Then for each $\varepsilon>0$ there exists $r>0$ such that, if $K_{R}(\gamma)<\varepsilon$ and $K_{R}(\mu)<\varepsilon$, then the distance between $\gamma$ and $\mu$ in the Kobayashi metric is greater than $r$.

Proof. Let $\nu:[0,1] \rightarrow R$ be a curve that connects $\gamma$ and $\mu$ so that $K_{R}(\nu)$ coincides with the distance between $\gamma$ and $\mu$. Let $\nu(0)=x_{0} \in \gamma$. By Corollary 2.2 it is enough to verify that $\gamma$ and $\gamma^{\prime}=\nu^{-1} \circ \mu \circ \nu$ generate noncommutative elements $[\gamma]$ and $\left[\gamma^{\prime}\right]$ in $\pi_{1}\left(R, x_{0}\right)$. Since the group $\pi_{1}\left(R, x_{0}\right)$ is free, $[\gamma]$ and $\left[\gamma^{\prime}\right]$ are commutative, iff they belong to a cyclic subgroup. This implies that $[\gamma]=[\nu]^{n}$ and $\left[\gamma^{\prime}\right]=[\nu]^{l}$ for a certain $[\nu] \in \pi_{1}\left(R, x_{0}\right)$. By Lemma 2.3 $k=l=1$. Hence $[\gamma]=\left[\gamma^{\prime}\right]$. Therefore $\gamma$ and $\mu$ must be homotopically equivalent. But this contradicts Lemma 2.4.

\section{LIMITING BEHAVIOR OF HYPERBOLIC METRIC}

From now on by $R$ we denote a connected hyperbolic component of $\Gamma_{0}^{*}$.

Lemma 3.1. Let $\alpha$ be a sequence of points in $\Delta^{*}$ that tends to zero. Suppose that for each $c \in \alpha$ the fiber $\Gamma_{c}$ is a hyperbolic Riemann surface. Then for a certain infinite subsequence $\beta=\left\{b_{j}\right\} \subset \alpha$ there exists a differential pseudometric $\alpha_{\beta}$ 
on $R$ such that $\alpha_{\beta}=\lim _{j \rightarrow \infty} k_{\Gamma_{b_{j}}}$. Moreover, $\alpha_{\beta}$ is a continuous pseudometric and the equality $\alpha_{\beta}(v)=0$ for a vector $v \in T R$ implies $\alpha_{\beta} \equiv 0$.

Proof. Let $\phi: \Delta \rightarrow R$ be a holomorphic embedding and $\phi(\Delta)=U$. It is easy to construct holomorphic embeddings $\phi_{j}: \Delta \rightarrow U_{j} \subset \Gamma_{b_{j}}$ so that $\phi_{j}(z) \rightarrow \phi(z)$ as $j \rightarrow \infty$ (e.g., see [Z]). Let $\nu_{z}$ denote the point $(z, d / d z) \in T \Delta$. We set $s_{z}^{j}=\phi_{j *}\left(\nu_{z}\right)$ and $s_{z}=\phi_{*}\left(\nu_{z}\right)$ (where $\phi_{j *}$ and $\phi_{*}$ are the induced mappings of the tangent bundles). Then $s_{z}^{j} \rightarrow s_{z}$ in topology of $T M$. Let $f_{j}: \Delta \rightarrow \Gamma_{b_{j}}$ be a universal holomorphic covering. Choose a connected component $V_{j}$ of $f_{j}^{-1}\left(U_{j}\right)$ and a holomorphic mapping $g_{j}: \Delta \rightarrow \Delta$ so that the restriction of $g_{j} \circ \phi_{j}^{-1} \circ f_{j}$ to $V_{j}$ is the identity mapping. One may suppose that $0 \in V_{j}$ and $g_{j}(0)=0$. Let $\tilde{s}_{z}^{j} \in T V_{j}$ belong to the inverse image of the vector $s_{z}^{j}$ under the mapping $f_{j *}$. Then $g_{j *}\left(\nu_{z}\right)=\tilde{s}_{z}^{j}$. On the other hand $g_{j *}\left(\nu_{z}\right)=g_{j}^{\prime}(z) \nu_{g_{j}(z)}$. Hence, taking into consideration the equalities $k_{\Delta}\left(\tilde{s}_{z}^{j}\right)=k_{\Gamma_{b_{j}}}\left(s_{z}^{j}\right)$ and $k_{\Delta}\left(\nu_{z}\right)=1 /\left(1-|z|^{2}\right)$, we have $k_{\Gamma_{b_{j}}}\left(s_{z}^{j}\right)=\left|g_{j}^{\prime}(z)\right| /\left(1-\left|g_{j}(z)\right|^{2}\right)$. Passing to a subsequence, if necessary, we suppose that $g_{j}(z) \rightarrow g(z)$ uniformly on compact subsets of $\Delta$. By Hurwitz's theorem either $g^{\prime}(z) \neq 0$ for every $z \in \Delta$ or $g^{\prime}(z) \equiv 0$ (in the last case $g(z) \equiv$ 0 , since $g(0)=0)$. Therefore $\lim _{j \rightarrow \infty} k_{\Gamma_{b_{j}}}\left(S_{z}^{j}\right)=\left|g^{\prime}(z)\right| /\left(1-|g(z)|^{2}\right)$. Let $s_{z}^{j}=\left(x_{j}(z), t_{j}(z)\right)$, where $x_{j}(z) \in U_{j}$ and $t_{j}(z) \in T_{x_{j}(z)} U_{j}$ (the notation, $s_{z}=$ $(x(z), t(z))$ has the same meaning). A sequence $\left\{v_{j} \mid v_{j}=\left(x\left(z_{j}\right), \lambda_{j} t_{j}\left(z_{j}\right)\right) ; \lambda \in\right.$ $C$ \} converges to $v=(x(z), \lambda t(z))$ in the topology of $T M$, iff $z_{j} \rightarrow z$ and $\lambda_{j} \rightarrow \lambda$. Hence $\lim _{j \rightarrow \infty} k_{\Gamma_{b_{j}}}\left(v_{j}\right)=\left|\lambda g^{\prime}(z)\right| /\left(1-|g(z)|^{2}\right)$ and a proper limiting pseudometric exists on $U$. Let $\left\{U^{j}\right\}$ be a cover on $R$ and each $U^{j}$ be an open disc. We can repeat the above construction of the limiting pseudometric for each $U^{j}$ instead of $U$. Application of the diagonal process completes the proof of the lemma.

Definition. Let $\beta=\left\{b_{j}\right\} \subset \Delta^{*}$ be a sequence that converges to zero, and let every fiber $\Gamma_{b_{j}}$ hyperbolic. We shall say that $\beta$ is an admissible sequence if there is a continuous differential pseudometric $a_{\beta}$ on $R$ such that $a_{\beta}=\lim _{j \rightarrow \infty} k_{\Gamma_{b_{j}}}$ and the quality $a_{\beta}(v)=0$ for a vector $v \in T R$ implies $a_{\beta} \equiv 0$. We will denote the corresponding integral pseudometric by $A_{\beta}$, and throughout the rest of the paper we will fix these notations $\beta, a_{\beta}$, and $A_{\beta}$ for the above objects.

Lemma 3.2. Suppose that the $a_{\beta}$ is a metric. Let $F=\left\{f_{j}\right\}$, where $f_{j}: \Delta \rightarrow \Gamma_{b_{j}}$ is a holomorphic universal covering with $f_{j}(0) \rightarrow x_{0} \in R$ as $j \rightarrow \infty$. Then there is a nonempty open subset $D \subset \Delta$ that contains 0 and a subsequence $F_{1} \subset F$ that converges to a mapping $f: D \rightarrow R$. Moreover

(i) $f: D \rightarrow R$ is an unramified covering.

(ii) $F$ transforms the metric $\left.k_{\Delta}\right|_{D}$ into the metric $a_{\beta}$.

Proof. First assume that $f$ exists and prove (ii). Let $z \in D, x=f(z)$, and $x_{j}=f_{j}(z)$. Then $x_{j} \rightarrow x$ as $j \rightarrow \infty$. Choose a sequence $\left\{v_{j} \mid v_{j} \in T_{x_{j}} \Gamma_{b_{j}}\right\}$ that converges to a nonzero vector $v \in T_{x} R$ in the topology of $T M$. Let $\tilde{v}_{j} \in T_{z} \Delta$ belong to the inverse image of $v_{j}$ under the mapping $f_{j *}$. Since $\beta$ is admissible, $k_{\Delta}\left(\tilde{v}_{j}\right)=k_{\Gamma_{b_{j}}}\left(v_{j}\right) \rightarrow a_{\beta}(v)$. Thus for every $j, k_{\Delta}\left(\tilde{v}_{j}\right)$ is less than a certain common constant. Hence we may suppose that there is the limiting vector $\tilde{v}$ for the sequence $\left\{\tilde{v}_{j}\right\}$. Clearly, $k_{\Delta}(\tilde{v})=a_{\beta}(v)$ and $f_{*}(\tilde{v})=v$. 
This implies (ii). Property (ii) means that, if $f$ exists, then it must be locally homeomorphic. Let $P$ be a sufficiently small neighborhood of $x_{0}$ such that $P$ is biholomorphically equivalent to a ball, and all $U_{j}=P \cap \Gamma_{b_{j}}$ and $U=\Gamma_{0} \cap P$ are discs. For every manifold $N$ we will denote by $B(y, r, N) \subset N$ the ball of radius $r$ in the metric $K_{N}$ with the center at $y$. Let $B\left(x, r, A_{\beta}, R\right) \subset R$ be the analogous ball in the metric $A_{\beta}$ with the center at $x$. Since $a_{\beta}$ is a metric, there exists $r>0$ such that $\overline{B\left(x_{0}, r, A_{\beta}, R\right)} \subset U$. Hence $\overline{B\left(f_{j}(0), r, \Gamma_{b_{j}}\right)} \subset U_{j}$, when $j$ is sufficiently large. The restriction of $f_{j}$ to $\widetilde{H}_{0}=B(0, r, \Delta)$ is a homeomorphism between $\widetilde{H}_{0}$ and $B\left(f_{j}(0), r, \Gamma_{b_{j}}\right)$. The family $\left\{\left.f_{j}\right|_{\widetilde{H}_{0}}: \widetilde{H}_{0} \rightarrow\right.$ $P\}$ is normal. Pick out a converging subsequence $F_{1} \subset F$ in this family. Let $f: \widetilde{H}_{0} \rightarrow B\left(x, r, A_{\beta}, R\right)$ be the limiting mapping. We have proved that $D \supset \widetilde{H}_{0}$, i.e., $D$ is not empty. Since $f$ is locally homeomorphic and each $\left.f_{j}\right|_{\widetilde{H}_{0}}$ is a homeomorphism, one can easily check that the limiting mapping $\left.f\right|_{\widetilde{H}_{0}}$ is also homeomorphism. Set $H=B\left(x_{0}, r / 3, A_{\beta}, R\right)$. Suppose there is a point $z \in D-\widetilde{H}_{0}$ with $y=f(z) \in H$. Let $H_{1}=B\left(y, 2 r / 3, A_{\beta}, R\right)$ and $\widetilde{H}_{1}=B(z, 2 r / 3, \Delta)$. Clearly $H_{1} \subset U$. Repeating the above arguments we can choose a subsequence $F_{2} \subset F_{1}$ so that the restriction $F_{2}$ to $\widetilde{H}_{1}$ converges to a homeomorphism $g: \widetilde{H}_{1} \rightarrow H_{1}$. For every subsequence $F_{3} \subset F_{1}-F_{2}$ that converges to a mapping $h: \widetilde{H}_{1} \rightarrow H_{1}$ we have $\left.g\right|_{\widetilde{H}_{1} \cap D}=\left.h\right|_{\widetilde{H}_{1} \cap D}=\left.f\right|_{\widetilde{H}_{1} \cap D}$. By the uniqueness theorem $h=g$. Thus one can take $F_{1}$ itself as $F_{2}$ and $D \supset \widetilde{H}_{1}$. Since $H_{1} \supset H, \widetilde{H}_{1}$ contains a disc $\widetilde{H}$ such that $\left.f\right|_{\widetilde{H}}: \widetilde{H} \rightarrow H$ is a homeomorphism and $\widetilde{H}_{0} \cap \widetilde{H}=\varnothing$ (indeed, $z \notin \widetilde{H}_{0}$ and the restriction of $f$ to $\widetilde{H}_{0}$ is also a homeomorphism). We can consider $H$ as a neighborhood of $x_{0}$. Of course, analogous arguments enable us to find such a neighborhood for every point $x \in F(D)$. Hence $f: D \rightarrow F(D)$ is an unramified covering. In particular, $f(D)$ is an open set.

To check the equality $R=f(D)$ it is enough to prove that the set $f(D)$ is closed in $R$. Let $x$ belong to the closure of $f(D)$ in $R$. Let $P^{\prime}$ be a sufficiently small neighborhood of $x$. Suppose that $P^{\prime}$ is biholomorphically equivalent to a ball, and $U^{\prime}=P^{\prime} \cap \Gamma_{0}$ and $\left\{U_{j}^{\prime}=P^{\prime} \cap \Gamma_{b_{j}}\right\}$ are discs. Choose $r>0$ with $\overline{B\left(x, r, A_{\beta}, R\right)} \subset U^{\prime}$. Then we can find a point $y \in f(D) \cap B\left(x, r / 3, A_{\beta}, R\right)$. As we have seen, in this case $f(D) \supset B\left(y, 2 r / 3, A_{\beta}, R\right)$. Hence, $x \in f(D)$, which is the desired conclusion.

Corollary 3.3. If the assumptions of Lemma 3.2 hold and $D=\Delta$, then $k_{R}=$ $\lim _{j \rightarrow \infty} k_{\Gamma_{b_{j}}}$.

Proof. We shall use the notation of the proof of Lemma 3.2. If $D=\Delta$, then $f: D \rightarrow R$ is a universal holomorphic covering and

$$
\lim _{j \rightarrow \infty} k_{\Gamma_{b_{j}}}\left(v_{j}\right)=\lim _{j \rightarrow \infty} k_{\Delta}\left(\tilde{v}_{j}\right)=k_{\Delta}(\tilde{v})=k_{D}(\tilde{v})=k_{R}(v) .
$$

\section{STEIN CASE}

From now on $M$ is a Stein surface, and we will use the same notations $R$, $\beta=\left\{b_{j}\right\}, a_{\beta}, f_{j}: \Delta \rightarrow \Gamma_{b_{j}}, F=\left\{f_{j}\right\}$ and $f: D \rightarrow R$ as in the preceding section. Let a Riemann surface $A$ be topologically an annulus. Denote the minimum of lengths of noncontractible loops in $A$ by $l(A)$. 
Proposition 4.1. To each number $t>0$ corresponds a positive number $r<1$ so that the assumptions:

(i) $L$ is a compact in $\Delta$;

(ii) $0 \in L$;

(iii) $\Delta-L$ is topologically an annulus;

(iv) $l(\Delta-L)<t$

imply that $L \subset \Delta_{r}$.

Proof. Assume that the contrary. Then for a certain $t$ and every $r<1$ there is compact $L_{r}$ that contains a point $z_{r}$ with $\left|z_{r}\right|>r$ and satisfies (i)-(iv). Clearly $l(L-\Delta)$ is greater than $2 K_{\Delta}\left(0, z_{r}\right)$. But $K_{\Delta}\left(0, z_{r}\right) \rightarrow \infty K_{\Delta}\left(0, z_{r}\right) \rightarrow \infty$ as $r \rightarrow 1$, and we have a contradiction with (iv).

According to [S] the Stein subvariety $R$ has a tubular neighborhood $V \subset M$ that is biholomorphically equivalent to a neighborhood of the zero section in the normal bundle to $R$ in $M$. Thus we have a holomorphic retraction $\tau: V \rightarrow R$. Let $Q$ be a region in $R$ with the compact closure. Then for a sufficiently small $\varepsilon$ and every $c \in \Delta_{\varepsilon}$ the restriction $\tau$ to $\tau^{-1}(Q) \cap \Gamma_{c}$ is a holomorphic unramified covering, whose multiplicity over $Q$ is equal to the multiplicity to zero of the function $\Phi$ on $R$.

Lemma 4.2. Let $\gamma$ be a loop in $R$ without points of self-intersection. Let $\left\{\bar{\phi}_{j} \mid \bar{\phi}_{j}: \bar{\Delta} \rightarrow \Gamma_{b_{j}}\right\}$ be continuous embeddings that are holomorphic on $\Delta$. Suppose that $\gamma_{j}=\bar{\phi}_{j}(\partial \Delta)$ belong to $\tau^{-1}(\gamma)$. Then $\gamma$ is contractible.

Proof. We shall consider the Stein manifold $M$ as a closed analytic submanifold in $C^{n}$ (e.g., see [GR]). Then each $\bar{\phi}_{j}$ has the following coordinate representation $\left.\phi_{j}(z)=\left(\bar{\phi}_{j 1}, \ldots, \bar{\phi}_{j n}\right)\right)$. Denote the restriction $\bar{\phi}_{j}$ to $\Delta$ by $\phi_{j}$, and let $\phi^{\prime}=\left(\phi_{j 1}^{\prime}, \ldots, \phi_{j n}^{\prime}\right)$ be the derivation of $\phi_{j}$. As usual we shall use the symbol $\left\|\phi_{j}^{\prime}(z)\right\|$ to denote the Euclidean length of the vector $\phi_{j}^{\prime}(z)$. Suppose that the functions $\left\|\phi_{j}^{\prime}\right\|$ converges to zero uniformly on compact subsets of $\Delta$. Then there exists a sequence of points $\left\{z_{j}\right\} \subset \Delta$ with $\left|z_{j}\right| \rightarrow 1$ that satisfies $\left\|\phi_{j}^{\prime}\left(z_{j}\right)\right\| \geq t /\left(1-\left|z_{j}\right|^{2}\right)$ for a certain positive $t$. Indeed, otherwise it is easy to show that the maximal Euclidean distance between the points of $\gamma_{j}$, tends to zero as $j \rightarrow \infty$. But $\gamma_{j}$ is close to $\tau\left(\gamma_{j}\right)$. This implies that $\gamma$ must be a constant mapping, and we have a contradiction. Put $\bar{\psi}_{j}=\phi_{j} \circ \mu_{j}$, where $\mu_{j}(z)=\left(z+z_{j}\right) /\left(1+\bar{z}_{j} z\right)$. Let $\psi_{j}=\left.\bar{\psi}_{j}\right|_{\Delta}$. The loop $\gamma$ belongs to a ball $B$ in $C^{n}$. Hence for an arbitrary large $j$ we have $\bar{\psi}_{j}(\partial \Delta) \subset B$. By the Maximum Principle $\bar{\psi}_{j}(\Delta) \subset B$. Therefore the family $\left\{\psi_{j}\right\}$ is normal. Passing to a subsequence, if necessary, we can suppose that $\left\{\psi_{j}\right\}$ converge to a mapping $\psi: \Delta \rightarrow \bar{R}$. Obviously, $\left\|\psi_{j}^{\prime}(0)\right\| \geq t$, and, therefore, $\psi$ is not constant. According to [Z, Lemma 2.2] $\psi(\Delta) \subset R$. Using a Möbius transformation again, if necessary, one may suppose that $\psi(0) \notin \gamma$. Choose an arbitrary small neighborhood $N$ of $\gamma$ in $R$ so that $N$ is topologically an annulus and $\psi(0) \notin N$. Then $N-\gamma$ consists of two components $N_{1}$ and $N_{2}$, which are also annuli. Let $\mu_{k}$ be the component of the boundary of $N_{k}$ other than $\gamma$. Obviously, $\psi_{j}(\Delta)$ must contain a component of either $\tau^{-1}\left(N_{1}\right) \cap \Gamma_{b_{j}}$ or $\tau^{-1}\left(N_{2}\right) \cap T_{b_{j}}$. Denote this component by $L_{j}$. Passing to a subsequence, we may suppose that $\tau\left(L_{j}\right)=N_{1}$ and $\left.\tau\right|_{L_{j}}$ is a $s$-sheeted unramified covering, where $s$ does not exceed the multiplicity of zero of the function $\Phi$ on $R$. Hence the Riemann surfaces $\left\{L_{j}\right\}$ are pairwise biholomorphically equivalent, and $l\left(L_{j}\right)=l\left(\psi_{j}^{-1}\left(L_{j}\right)\right)$ does not 
depend on $j$. Since $0 \notin \psi_{j}^{-1}\left(L_{j}\right)$, we see by Proposition 4.1 that there is a positive $r<1$ such that $\Delta-\psi_{j}^{-1}\left(L_{j}\right) \subset \Delta_{r}$. Hence $\mu_{1} \subset \psi\left(\Delta_{r}\right)$. This implies that $\mu_{1}$ is contractible, and, therefore, $\gamma$ is also contractible.

Lemma 4.3. The pseudometric $a_{\beta}$ generated by an admissible sequence $\beta$ is $a$ metric on $R$ in the case when $R$ is different from $\Delta, \Delta^{*}$, or an annulus.

Proof. Let $\gamma_{1}, \ldots, \gamma_{k}$ be disjoint noncontractible loops in $R$ without points of self-intersection such that they are not pairwise homotopically equivalent, for each $i$ the set $R-\gamma_{i}$ is not connected, and every $\gamma_{i}$ is a component of the boundary of a compact $L \subset R$. Let $L_{j}$ be a component of $\tau^{-1}(L) \cap \Gamma_{b_{j}}$. One may suppose that $\left.\tau\right|_{L_{j}}: L_{j} \rightarrow L$ is a $s$-sheeted unramified covering for all $j$. Let $\left\{\gamma_{i j}^{l} \mid l=1, \ldots, l_{i j} \leq s\right\}$ be the components of $\tau^{-1}\left(\gamma_{i}\right) \cap L_{j}$. If $R$ has a positive genus, we can suppose that $L$ contains a loop $\mu$ without points of self-intersection so that $L-\mu$ is connected and $\mu \cap \bigcup_{j=1}^{k} \gamma_{j}=\varnothing$. In this case we denote one of the components of $\gamma^{-1}(\mu) \cap \Gamma_{b_{j}}$ by $\mu_{j}$. Assume, to reach a contradiction, that $a_{\beta} \equiv 0$. Then $K_{\Gamma_{b_{j}}}\left(\gamma_{i j}^{l}\right), K_{\Gamma_{b_{j}}}\left(\mu_{j}\right) \rightarrow 0$ as $j \rightarrow \infty$ and the distance between each pair of these loops in the Kobayashi metric on $\Gamma_{b_{j}}$ also tends to zero. By Lemma 2.5 all of these loops must be homotopically equivalent. Since $\left.\tau\right|_{L_{j}}: L_{j} \rightarrow L$ is an unramified covering, $L_{j}-\mu_{j}$ is connected. Hence by Lemma $2.4 \mu_{j}$ cannot be homotopically equivalent to any component of the boundary of $L_{j}$, or in other words, to any $\gamma_{i j}^{l}$. Therefore it remains to consider the case when $R$ is biholomorphically equivalent to a region in $C$. Then under the assumptions of the lemma one may suppose that $k \geq 3$. Thus we have, at least, three loops $\gamma_{1}, \gamma_{2}$, and $\gamma_{3}$. By Lemma 2.5 there is a region $U_{j} \subset \Gamma_{b_{j}}$ such that $\partial U_{j}=\gamma_{1 j}^{1} \cup \gamma_{2 j}^{1}$ and $U_{j}$ is topologically an annulus. Note that $U_{j}$ does not belong to $L_{j}$ (otherwise, using Lemmas 2.3 and 2.4 it is easy to show that $\gamma_{1}$ and $\gamma_{2}$ are homotopically equivalent). Moreover, since the component of $\Gamma_{b_{j}}-L_{j}$ whose boundary contains $\gamma_{3 j}^{1}$ is different from a disc according to Lemma 4.2, $U_{j}$ does not contain $L_{j}$. Hence $U_{j}$ is a component of $\Gamma_{b_{j}}-L_{j}$. Taking $\gamma_{3 j}^{1}$ instead of $\gamma_{2 j}^{1}$ we can construct a component $V_{j}$ of $\Gamma_{b_{j}}-L_{j}$ so that $\partial V_{j}=\gamma_{1 j}^{1} \cup \gamma_{3 j}^{1}$ and $V_{j}$ is topologically an annulus. Since $\partial V_{j} \cap \partial U_{j}=\gamma_{1 j}^{1}, V_{j}=U_{j}$. Then $\partial U_{j}=\partial V_{j}$, and this leads to a contradiction. Therefore $a_{\beta}$ is not trivial. By Lemma $3.1 a_{\beta}(v) \neq 0$ for each $v \in T R$. This completes the proof of the lemma.

Lemma 4.4. Let $M$ be a Stein surface and let $D$ be the same as in Lemma 3.2. Then $D$ is simply connected.

Proof. Assume that $D$ is not simply connected. Then there is a couple of discs $d$ and $d^{\prime}$ such that $\bar{d} \subset \Delta, d^{\prime} \subset d, d$ does not belong to $D$, and $\bar{d}-d^{\prime} \subset D$. We again consider $M$ as a submanifold in $C^{n}$. The set $f\left(\bar{d}-d^{\prime}\right)$ belongs to a certain ball in $C^{n}$. Same arguments as in Lemma 4.2 show that the family $\left\{\left.f_{j}\right|_{d}\right\}$ is normal. Let $\tilde{f}: d \rightarrow \bar{R}$ be a limiting mapping. This mapping is unique, since it coincides with $f$ on $d-d^{\prime}$. In particular, it is nonconstant. The set $f(d)$ does not contain singular points of $\Gamma_{0}$, because otherwise $f_{j}(d)$ must intersect $\Gamma_{0}$ for an arbitrary large $j[\mathrm{Z}]$. Hence $\tilde{f}(d) \subset R$, i.e., $d \subset D$. But this contradicts our assumption. 
Corollary 4.5. Lemma 4.2 holds without the condition that $\gamma$ has no point of self-intersection.

\section{PROOF OF THE MAIN THEOREM}

We keep the same notation $R, \beta, a_{\beta}, F=\left\{f_{j}\right\}, f: D \rightarrow R$ as in the preceding section. By Lemmas 3.2, 4.3, and 4.4 we suppose that the family $F$ converges to the mapping $f: D \rightarrow R$ on a nonempty simply connected region $D \subset \Delta$ with $0 \in D$, and $f$ is an unramified covering, which transforms the metric $\left.k_{\Delta}\right|_{D}$ into the metric $a_{\beta}$. Let $G_{j}$ be the Fuchsian group such that $f_{j}(z)=f_{j}\left(z^{\prime}\right)$ iff $z^{\prime}=g(z)$ for a certain $g \in G_{j}$. We say that a Möbius transformation $h$ is limiting for $\left\{G_{j}\right\}$, if there is a sequence $\left\{g_{j} \mid g_{j} \in G_{j}\right\}$ that converges to $h$ uniformly on compact subsets of $\Delta$. Let $G$ be the group of holomorphic one-to-one mappings $D$ to $D$ such that $f(z)=f\left(z^{\prime}\right)$, if $z^{\prime}=$ $g(z)$ for a certain $g \in G$.

Lemma 5.1. The set $H$ of limiting Möbius transformations is a subgroup of $G$ of finite index.

Proof. By construction, $H$ is a group and for each pair $z, z^{\prime} \in D$ the equality $h(z)=z^{\prime}$ for an element $h \in H$ implies $f(z)=f\left(z^{\prime}\right)$. Hence $H \subset G$. As in the preceding section $\tau: V \rightarrow R$ is a holomorphic retraction of a Stein neighborhood $V$ of $R$. Consider all the loops $\{\gamma: \partial \Delta \rightarrow R\}$ such that $\gamma(1)=$ $f(0)$ and for an arbitrary large $j$ there is a loop $\gamma_{j}$ in $\Gamma_{b_{j}}$ with $\gamma_{j}(1)=$ $f_{j}(0)$ and $\gamma=\tau \circ \gamma_{j}$. These loops generate a subsgroup $H_{1}$ of finite index in $\pi_{1}(R, f(0))$. This index does not exceed the multiplicity of zero of the function $\Phi$ on $R$. Since $\pi_{1} \cong G$, one can consider $H_{1}$ as a subgroup in $G$ as well. Let $\gamma$ be a loop in $R$ with $[\gamma] \in H_{1}$ and $\left\{\gamma_{j} \in \Gamma_{b_{j}}\right\}$ be the corresponding loops, which converge to $\gamma$ uniformly. Consider the mappings $\nu_{j}: \mathbf{R} \rightarrow \Delta$ and $\nu: \mathbf{R} \rightarrow D$ such that $f_{j} \circ \nu_{j}(t)=\gamma_{j}\left(e^{2 \pi i t}\right), f \circ \nu(t)=\gamma\left(e^{2 \pi i t}\right)$, and $\nu(0)=\nu_{j}(0)=0$. Since $\gamma_{j} \rightarrow \gamma$ and $f_{j} \rightarrow f$, one can see that $\nu_{j} \rightarrow \nu$ uniformly. By $\tilde{\gamma}_{j}$ and $\tilde{\gamma}$ we will denote the elements of the Fuchsian groups $G_{j}$ and $G$ that correspond $\left[\gamma_{j}\right]$ and $[\gamma]$ respectively. Clearly, $\tilde{\gamma}_{j}^{k}(t)=\nu_{j}(t+k)$ and $\tilde{\gamma}^{k}(t)=\nu(t+k)$ for each integer $k$. This means $\tilde{\gamma}_{j} \rightarrow \tilde{\gamma}$ as $j \rightarrow \infty$. Hence $H_{1} \subset H$ and $H$ is a subgroup of $G$ of finite index.

Let $\widetilde{R} \rightarrow R$ be an unramified covering that corresponds to the subgroup $H \subset$ $\pi_{1}(R)$. Then, since $D$ is simply connected, the mapping $\tilde{f}: D \rightarrow D / H \cong \widetilde{R}$ is a universal holomorphic covering. Recall that by the hypotheses of Main Theorem $G$ is isomorphic to a Fuchsian group of the first kind $G^{\prime}$, acting on $\Delta$. More precisely, there is a biholomorphic mapping $\varphi: \Delta \rightarrow D$ such that $\varphi$ generates isomorphism between $G$ and $G^{\prime}$. Therefore $H$ is isomorphic to a subgroup $H^{\prime}$ of finite order in $G^{\prime}$. Hence $H^{\prime}$ is a Fuchsian group of the first kind as well. According to [G, $\S 3$, Lemma 3] it is easy to check now that, since the closure of the orbits $\left\{h^{\prime}(0) \mid h^{\prime} \in H^{\prime}\right\}$ coincides with $\partial \Delta$, the closure of orbits $\{h(0) \mid h \in H)$ must coincide with $\partial D$. Assume that $z$ is a point of $\partial D \cap \Delta$. Choose an arbitrary small neighborhood $U$ of $z$ and element $\tilde{v}, \tilde{\eta} \in H$ so that $\tilde{\nu}(0)$ and $\tilde{\eta} \circ \tilde{\nu}(0) \in U \cap D$. Let $\tilde{\mu}, \tilde{\gamma} \in H$ be noncommutative elements. Then $\tilde{\eta}, \tilde{\nu}^{-1}$, $\tilde{\gamma} \circ \tilde{\nu}^{-1}, \tilde{\mu} \circ \tilde{\nu}^{-1}$ cannot belong to a cyclic subgroup of $H$. Hence one of the pairs $\tilde{\eta}$ and $\tilde{\nu}^{-1}, \tilde{\eta}$ and $\tilde{\gamma} \circ \tilde{\nu}^{-1}$ or $\tilde{\eta}$ and $\tilde{\mu} \circ \tilde{\nu}^{-1}$ are not commutative. Consider the corresponding noncommutative pair of elements in $G_{j}$ for a sufficiently large 
$j$. Put $z^{\prime}=\tilde{\nu}(0)$. Application of Lemma 2.1 to the above pair and the point $z^{\prime}$ leads to a contradiction. Thus $D=\Delta$ and by Corollary $3.3 k_{R}=\lim _{j \rightarrow \infty} k_{\Gamma_{b_{j}}}$. This implies immediately that for every sequence $\left\{b_{j}\right\} \subset \Delta^{*}$ with hyperbolic fibers $\left\{\Gamma_{b_{j}}\right\}$ and $b_{j} \rightarrow 0 \quad k_{R}=\lim _{j \rightarrow \infty} k_{\Gamma_{b_{j}}}$. The last thing we need to confirm is that if there exists a sequence $\left\{b_{j}\right\} \rightarrow 0$ with nonhyperbolic fibers $\left\{\Gamma_{b_{j}}\right\}$ then $R$ cannot be hyperbolic. Assume that such a sequence exists. Then $\Gamma_{b_{j}}$ is biholomorphically equivalent to $C$ or $C^{*}$. Hence $R$ has no handle, for if it had, then all of the fibers $\Gamma_{b_{j}}$ would have handles as well for sufficiently large $j$. Since a Fuchsian group of the first kind corresponds to the Riemann surface $R, R$ is different from $\Delta, \Delta^{*}$ or an annulus. Thus $\pi_{1}(R)$ has, at least, two generators $\left[\gamma_{1}\right]$ and $\left[\gamma_{2}\right]$. One may suppose that the loops $\gamma_{1}$ and $\gamma_{2}$ have no points of self-intersection. Note that the proof of Lemma 4.2 does not use the assumption that $\left\{\Gamma_{b_{j}}\right\}$ are hyperbolic, i.e., it remains true without this assumption. Thus, since $\Gamma_{b_{j}}$ is biholomorphically equivalent to $C$ or $C^{*}$ either $\gamma_{1}^{k}$ or $\gamma_{2}^{k}$ must be approximated by contractible loops in $\left\{\Gamma_{b_{j}}\right\}$ for a certain integer $k$. This contradicts Lemma 4.2. Hence there is no sequence $\left\{b_{j}\right\} \rightarrow 0$ with nonhyperbolic fibers $\left\{\Gamma_{b_{j}}\right\}$. The main theorem is proved.

\section{REFERENCES}

[A] W. Abikoff, The real analytic theory of Teichmüller space, Lecture Notes in Math., vol. 820, Springer-Verlag, Berlin and New York, 1980.

[B] L. Bers, Uniformization, moduli, and Kleinian groups, Bull. London Math. Soc. 4 (1972), 257-300.

[G] G. M. Goluzin, Geometric theory of functions of a complex variable, Amer. Math. Soc., Providence, R.I., 1969.

[GR] R. G. Gunning and H. Rossi, Analytic functions of several complex variables, Prentice-Hall, Englewood Cliffs, N.J., 1965.

[Ka] Sh. Kaliman, Polynomials on $C^{2}$ with isomorphic generic fibers, Soviet Math. Dokl. 33 (1986), 600-603.

[Ko] S. Kobayashi, Hyperbolic manifolds and holomorphic mappings, Marcel Dekker, New York, 1970.

[PS] E. A. Poletsky and B. V. Shabat, Invariant metrics, Itogi Nauki i Tekhniki (Modern Problems of Mathematics, Fundamental Directions), VINITI, Moscow, 1986, pp. 73-125. (Russian)

[R] H. Royden, Remarks on Kobayashi metric, Several Complex Variables II (Proc. Internat. Conf., College Park, Md., 1970), Lecture Notes in Math., vol. 185, Springer-Verlag, 1971, pp. 125-137.

[S] Y.-T. Siu, Every Stein subvariety admits a Stein neighborhood, Invent. Math. 38 (1976), 89-100.

[Z] M. G. Zaidenberg, On hyperbolic embeddings of complements of divisors and the limiting behavior of the Kobayashi-Royden metric, Math. USSR-Sb. 55 (1986), 55-70.

Department of Mathematics, Wayne State University, Detroit, Michigan 48202

Current address: Department of Mathematics \& Computer Science, University of Miami, Coral Gables, Florida 33124 